\title{
Are we Under Utilizing the Medical Therapies in Preventing the Complications in Peripheral Arterial Disease?
}

\section{Ramakrishna PK}

Department of Vascular Surgery, Nizam's Institute of Medical sciences, Panjagutta, Hyderabad, Telangana, India

*Corresponding author: Ramakrishna PK, Department of Vascular Surgery, Nizam's Institute of Medical sciences, Panjagutta, Hyderabad, 500082, Telangana, India, Tel: +91 23317115, +91 9848050243; E-mail: pinjala@hotmail.com

Received date: April 13, 2017; Accepted date: May 08, 2017; Published date: May 15, 2017

Copyright: () 2017 Ramakrishna PK. This is an open-access article distributed under the terms of the Creative Commons Attribution License, which permits unrestricted use, distribution, and reproduction in any medium, provided the original author and source are credited.

Lower limb Peripheral Artery Disease (PAD) is the third most common cause of atherosclerotic cardiovascular morbidity after ischemic heart disease and stroke. Lower limb arterial disease is known to affect the quality of life of elderly people and it is associated increased cardiovascular complications and mortality. Smoking, Diabetes, Hypertension and hyper-lipidemic are the risk factors for peripheral vascular disease and the associated complications. The first global analysis of the lower limb arterial disease found that more than a quarter of a billion people in the world have the disease and poorer countries are disproportionately affected. Like many other life style diseases PAD incidence is also increasing during the past few decades. According to Fowkes $\mathrm{G}$ from the centre for population health sciences at the University of Edinburgh in Scotland, the global prevalence of PAD increased by $24 \%$ between $2000-2010$, that is from 164 million to 202 million [1]. In this 21st century, peripheral artery disease has already become a global problem and we are looking for better solutions and preventive measures. Governments, non-governmental organizations, and the private sector in Low and middle income countries are evaluating the social and economic consequences, and assessing the best strategies for implementation of optimum treatments and preventive measures. Interventional therapies for peripheral arterial disease patients with different types of stents and balloons seem to be attractive but difficult to reach the large population. Adequate medical therapies such as cessation of smoking, diabetes care, antihypertensive medications, Statin therapies, antiplatelet therapies, anticoagulants and supervised exercise therapies can reach the people even in the remote places and helpful in preventing the worsening of the PAD and reduce the cardiovascular complications, amputations and mortality.

In the Edinburgh artery study of the general population found that the prevalence of peripheral arterial disease was high in the asymptomatic or symptomatic form. The prevalence of intermittent claudication was $4.5 \%$. Major asymptomatic disease causing a significant impairment of blood flow occurred in $8.0 \%$. A further $16.6 \%$ had criteria considered abnormal in clinical practice: $9.0 \%$ had ABPI less than 0.9 and $7.6 \%$ had reactive hyperaemia pressure reduction greater than $20 \%$. Intermittent claudication was equally common in both sexes. Subjects with major asymptomatic lower limb arterial disease had more evidence of heart disease than in the normal population [2]. In PAD patients undergoing vascular surgery the longterm prognosis is significantly worse than that for the patients with Coronary Artery Disease (CAD). The patients who underwent vascular surgery received less cardiac medication and risk factor management than CAD patients. The cerebro-cardiovascular events are the major cause of late death in these patients after the vascular surgery in the long term [3].
Atherosclerosis affects numerous vascular beds to varying degrees. So, it is considered to be systemic disease. In patients with lower limb arterial disease, the prevalence of CAD was $46 \%$ to $71 \%[4,5]$. The prognosis after vascular surgery in these patients is predominantly influenced by the underlying CAD in the early post-operative period and long term [6]. The cardiac death accounts for $40 \%$ in the first 30 day post-operative mortality and $6 \%$ to $10 \%$ in the 1 -year mortality of vascular surgery patients [7].

We can improve the results in patients with PAD requiring surgery, with better evaluation of the risk factors and aggressive therapy of atherosclerotic risk factors. The secondary prevention measures in PAD patients are similar to the measures applied for patients with CAD. Aspirin is the antiplatelet drug and a better choice because of its low cost and the high incidence of CAD in PAD patients. It is interesting note that in the Physician Health Study of aspirin $325 \mathrm{mg}$ every other day decreased the incidence of peripheral artery surgery [8].

Clopidogrel is used in patients who are allergic or intolerant to aspirin. In the CAPRIE trial (Clopidogrel versus Aspirin in Patients at Risk of Ischemic Events) clopidogrel (75 mg/day) showed significant advantage over aspirin (325 mg/day) in preventing stroke, myocardial infarction and PAD in 19,185 patients after a recent stroke, myocardial infarction or PAD (The annual event rate was $5.3 \%$ vs. $5.8 \%$ ) [9-12]. Cilostazol which is a phosphodiesterase inhibitor was approved by the FDA for the treatment of Intermittent Claudication. It is vasodilator and suppresses platelet aggregation. In a meta-analysis of 8 placebocontrolled randomized trials 2,702 patients with stable moderate to severe claudication were included. They were treated with cilostazol $100 \mathrm{mg}$ twice daily for 12-24 weeks. It increased the maximal walking distance and pain-free walking distance by $50 \%$ and $67 \%$. These effects were seen as early as a 1 month. The evidence of benefit supported the therapeutic trial for 3-6 months of cilostazol $100 \mathrm{mg}$ twice daily to improve symptoms and increase walking distance in patients with lifestyle-limiting claudication. These are patients where antiplatelet agents and exercise rehabilitation are not helpful and revascularization cannot be done or refused by the patients. Prasugrel was introduced with lot of enthusiasm among patients with unstable angina or myocardial infarction without ST-segment elevation. But, prasugrel did not significantly reduce the frequency of the primary end point, when compared to the clopidogrel. The risks of bleeding were similar with Prasugrel and clopidogrel. The new antiplatelet drugs ticagrelol and prasugrel were promising options for treatment of PAD. Ticagrelor, is an inhibitor of platelet P2Y12 receptor, it has shown benefit acute coronary syndromes patients and stable coronary artery disease patients. Ticagrelor was not shown to be superior to clopidogrel for the reduction of cardiovascular events in lower limb arterial disease 
Page 2 of 2

patients. Major bleeding similarly among the patients receiving clopidogrel and ticagrelor.

Use of Statins in vascular surgery patients decreased the progression and even regressed growth of the atherosclerotic plaques. In patients with atherosclerosis of the lower limbs who have frequently concurrent coronary or cerebrovascular disease, use of statins resulted in decreased incidence of heart attacks and stroke. Statins are associated with pleiotropic benefits, such as anti-inflammatory effects which contribute to the additional beneficial effects and reduction in cardiovascular death. Many trials during the last few years showed that the statins improved pain-free walking distance, ankle brachial index and treadmill exercise time. So, the current guidelines for cholesterol reduction in PAD patients adhere to the same indications as for other cardiovascular diseases and strongly recommend the use of statins in these lower limb arterial disease patients [13].

Now, it is well understood that medical therapies are as important as the interventions in the vascular diseases. Earlier studies have documented under treatment of atherosclerotic risk factors among coronary artery disease patients. We realize that the atherosclerotic risk factor therapy and management may be even lower in PAD patients. If the strong association between PAD and cardiovascular mortality is not appreciated, it may also result in under treatment. Atherosclerotic risk factors are more aggressively treated among CAD patients than among PAD patients. PAD patients do not take the risk factor management advices sincerely. PAD patients fail to recall a physician's advice to do exercise, do not recall dietary advice for hypercholesterolemia, and more likely to fail taking aspirin or warfarin [14]. If there are deficiencies in the physician knowledge and attitudes that can also contribute to lower rates of atherosclerotic risk factor reduction for PAD patients. If can improve these deficiencies one can certainly reduce the excess cardiovascular morbidity and mortality associated with PAD [15]. In the PAD Awareness, Risk, and Treatment: New Resources for Survival (PARTNERS) program, PAD was identified in $29 \%$ of the participants out of 6,979 older patients in 350 general medicine practices across the United States. It is important note that compared to the participants with CVD diseases other than PAD, PAD participants in PARTNERS were under treated for hypertension and hyperlipidemia and received antiplatelet therapy less often. In adequate treatment can lead to high incidence of CVD morbidity and mortality in PAD patients [16]. Criqui et al. found that after the exclusion of participant with history of cardiovascular disease at base line, the relative cardiovascular risks among those with largevessel peripheral arterial disease remained significantly elevated. The secondary analyses by them showed a 15 -fold increase in rates of mortality due to cardiovascular disease and coronary heart disease among participants with large-vessel peripheral arterial disease which is severe and symptomatic [17].

Peripheral arterial disease in the lower limb is common in elderly people, these are at high risk of limb loss and higher mortality due to cardiovascular complications. There is urgent need for adequate medical therapies to prevent the complications and reduce the mortality. Adequate management of diabetes, hypertension, hyperlipidemia, appropriate use of antiplatelet drugs, anticoagulants, hemorheological agents along with cessation of smoking will be essential to prevent the morbidity and mortality vascular surgical patients [18].

\section{References}

1. Fowkes FGR (2013) Comparison of global estimates of prevalence and risk factors for peripheral artery disease in 2000 and 2010: a systematic review and analysis. The Lancet 382: 1329-1340.

2. Fowkes FGR, Housley E, Cawood EHH, Macintyre CCA, Ruckley CV, et al. (1991) Edinburgh Artery Study prevalence of asymptomatic and symptomatic peripheral arterial disease in the general population. Int J Epidemiol 20: 384-392.

3. Gijs MJMW, Olaf S, Sanne EH, Michel C, Radosav V, et al. (2008) LongTerm Prognosis of Patients With Peripheral Arterial Disease A Comparison in Patients With Coronary Artery Disease. J Am Coll Cardiol 51: 1588-1596.

4. Sukhija R, Aronow WS, Yalamanchili K, Sinha N, Babu S (2004) Prevalence of coronary artery disease, lower extremity peripheral arterial disease, and cerebrovascular disease in 110 men with an abdominal aortic aneurysm. Am J Cardiol 94: 1358-1359.

5. Dieter RS, Tomasson J, Gudjonsson T (2003) Lower extremity peripheral arterial disease in hospitalized patients with coronary artery disease. Vasc Med 8: 2336.

6. Back MR, Leo F, Cuthbertson D, Johnson BL, Shamesmd ML, et al. (2004) Long-term survival after vascular surgery: specific influence of cardiac factors and implications for preoperative evaluation. J Vasc Surg 40: 752-760.

7. McFalls EO, Ward HB, Santilli S, Scheftel M, Chesler E, et al. (1998) The influence of perioperative myocardial infarction on long term prognosis following elective vascular surgery. Chest 113: 681-686.

8. Goldhaber SZ, Manson JE, Stampfer MJ (1992) Low-dose aspirin and subsequent peripheral arterial surgery in the Physicians' Health Study. Lancet 340: 143-145.

9. CAPRIE Steering Committee (1996) A randomized, blinded, trial of clopidogrel versus aspirin in patients at risk of ischemic events (CAPRIE). Lancet 348: 1329-1339.

10. Reilly MP, Mohler ER (2001) Cilostazol: treatment of intermittent claudication. Ann Pharmacother 35: 48-56.

11. Thompson PD, Zimet R, Forbes WP (2002) Meta-analysis of results from eight randomized, placebo-controlled trials on the effect of cilostazol on patients with intermittent claudication. Am J Cardiol 90: 1314-1319.

12. Clagett GP, Sobel M, Jackson MR (2004) Antithrombotic therapy in peripheral arterial occlusive disease. The seventh ACCP conference on antithrombotic and thrombolytic therapy. Chest 126: S609-S626.

13. Markel A (2015) Statins and peripheral arterial disease. Int Angiol 34: 416-427.

14. McDermott MM, Mehta S, Ahn H, Greenland P (1997) Atherosclerotic Risk Factors Are Less Intensively Treated in Patients with Peripheral Arterial Disease Than in Patients with Coronary Artery Disease. J Gen Intern Med 12: 209-215.

15. McDermott MM, Hahn EA, Greenland P, Cella D, Ockene JK, et al. (2002) Atherosclerotic risk factor reduction in peripheral arterial disease: results of a national physician survey. J Gen Intern Med 17: 895-904.

16. Hirsch AT, Criqui MH, Treat-Jacobson D (2001) Peripheral arterial disease detection, awareness, and treatment in primary care. JAMA 286: 1317-1324.

17. Criqui MH, Langer RD, Fronek A (1992) Mortality over a period of 10 years in patients with peripheral arterial disease. N Engl J Med 326: 381-386.

18. Brian DC, Christian CA, Diane F, Neal DF, Patricia H, et al. (2015) Smoking and Mortality-Beyond Established Causes. N Engl J Med 372: 631-640. 